Cahiers $d u$ MONDE RUSSE

\section{Cahiers du monde russe}

Russie - Empire russe - Union soviétique et États indépendants

$62 / 4 \mid 2021$

Varia

\title{
Sheila FITZPATRICK, White Russians, Red Peril. A Cold War History of Migration to Australia
}

\section{Catherine Gousseff}

\section{(2penEdition}

\section{Journals}

Édition électronique

URL : https://journals.openedition.org/monderusse/12850

DOI : 10.4000/monderusse. 12850

ISSN : $1777-5388$

\section{Éditeur}

Éditions de l'EHESS

\section{Édition imprimée}

Date de publication : 1 décembre 2021

Pagination : 724-727

ISBN : 978-2-7132-2895-7

ISSN : $1252-6576$

Référence électronique

Catherine Gousseff, "Sheila FITZPATRICK, White Russians, Red Peril. A Cold War History of Migration to Australia », Cahiers du monde russe [En ligne], 62/4 | 2021, mis en ligne le 01 décembre 2021, consulté le 03 septembre 2022. URL : http://journals.openedition.org/monderusse/12850 ; DOI : https:// doi.org/10.4000/monderusse. 12850

Ce document a été généré automatiquement le 3 septembre 2022.

Tous droits réservés 


\title{
Sheila FITZPATRICK, White Russians, Red Peril. A Cold War History of Migration to Australia
}

\author{
Catherine Gousseff
}

\section{RÉFÉRENCE}

Sheila FITZPATRICK, White Russians, Red Peril. A Cold War History of Migration to Australia, London - New York : Routledge, 2021, 368 p.

1 L'originalité de cet ouvrage, au vu de l'abondante historiographie consacrée à l'émigration russe, dite "blanche », est de se situer à la croisée de champs encore peu ou mal explorés, qu'il s'agisse des destinées de l'émigration après la seconde guerre mondiale, de la configuration et des déplacements des colonies russes extra-européennes de Mandchourie et de Shanghai, ou encore de la rencontre, à questionner, entre vieux émigrés des années 1920 et nouveaux réfugiés soviétiques de l'après-guerre. Tous ces éclairages, qui font la riche matière de White Russians, Red Peril, soulignent la spécificité de la migration russe vers l'Australie. Cette terre de refuge tardive (le pays ne comptait guère plus de 5000 Russes dans l'entre-deux-guerres) accueillit, à partir de la fin des années 1940, des antibolcheviks chassés par la révolution chinoise, des Russes blancs et des Soviétiques en provenance des camps de personnes déplacées d'Allemagne et d'Autriche. Ces deux grandes migrations, d'Europe et d'Asie, qui convergèrent vers l'Australie, avaient en commun d'être identifiées comme étant celles de Russes et/ou de Soviétiques, de représenter, pour plus de la moitié des migrants au moins, un nouveau et ultime déplacement, après plus de vingt ans d'exil. Le meilleur passeport qui garantissait l'accueil australien était leur anticommunisme, revendiqué ou inscrit dans l'implicite de leur errance. Cette Cold War history of migration to Australia décrit, dans l'examen du cas russe, le grand changement de paradigme opéré dans l'appréhension occidentale du réfugié d'après-guerre. $\mathrm{Si}$, dans les premières années, celui-ci fut scruté dans son rapport au nazisme (victime ou collaborateur, et les Russes furent plus nombreux dans la seconde 
catégorie), l'obsession croissante du péril rouge à l'Ouest entraîna une tolérance de fait à l'égard des anciens complices d'Hitler. Mais le contexte de guerre froide ne constitue que la part ultime de cette rétrospective, même si l'ouvrage s'ouvre sur cette période pour en souligner la complexité et les questionnements qui vont guider l'enquête sur les Russes «blancs ». Le cœur de White Russians, Red Peril, se concentre en effet sur l'histoire des collectivités en question, dans la multitude des parcours, des appartenances identitaires et la manière dont s'est structuré le lien communautaire depuis les grands départs de Russie. Pour saisir les traits communs, et ce qui les distingue, des colonies russes d'Europe et d'Asie, sans pour autant niveler l'irréductible diversité des groupes, l'historienne conduit sa rétrospective en l'émaillant de nombreux récits de vie collectés auprès des descendants d'émigrés en Australie. Ils donnent chair à cette vaste histoire, par ailleurs retracée à l'appui de sources très variées, dont divers services de surveillance des pays d'accueil (police de Shangaï, intelligence service d'Australie) qui fournissent un regard extérieur, essentiellement politique, sur les Russes blancs. Les points d'observation sont donc multiples pour retracer successivement la formation et l'évolution de ces collectivités géographiquement dispersées. Il s'agit d'abord d'en esquisser les traits caractéristiques, dont beaucoup sont partagés, indépendamment des lieux d'implantations : les colonies russes d'Europe et d'Asie ont, pour partie, incarné les différentes destinées des anciennes unités combattantes de l'armée blanche. S'agissant de l'Europe, S. Fitzpatrick désigne surtout, sans toutefois toujours le spécifier, les implantations russes de Bulgarie et de Yougoslavie où les vétérans de l'armée Wrangel avaient été acheminés, après les évacuations de Russie du sud et leur transit par le Bosphore. Harbin, en Mandchourie, accueillit de nombreux contingents ayant effectué leur retraite vers l'Extrême-Orient après avoir combattu dans l'Oural, la Sibérie. À Harbin, souligne l'auteure, la présence massive des cosaques a fait d'eux le groupe social le plus organisé et le plus important d'une colonie russe, par ailleurs très diversifiée, comptant, notamment, de nombreux Soviétiques. À Shanghai, la colonie russe antibolchévique prit forme avec l'arrivée d'une flottille venue de Vladivostok via la Corée sous le commandement de l'amiral Stark au début des années 1920. Bien que dans chacun des lieux, les venues parallèles ou ultérieures diversifièrent considérablement les communautés, l'empreinte des vétérans de l'armée blanche fut partout forte, que l'on trouve aussi bien dans le dynamisme des unions militaires, parfois détaillées dans les subtiles nuances des différentes traditions de corps, que dans leur présence dans la presse ou encore leur rôle à la tête des offices émigrés. Sous diverses appellations, ces administrations dédiées à l'enregistrement et à la délivrance de certificats d'identité allaient se révéler stratégiques, pour ficher, exclure, dès lors que leurs dirigeants devinrent des figures de proue du fascisme russe. Au sein de la sphère d'activité des Russes blancs, entre scoutisme, associations philanthropiques et autres, Sheila Fitzpatrick accorde une attention soutenue au rôle de l'Église orthodoxe, et, plus précisément, à celui de l'Église russe Hors frontières, issue du premier schisme de l'émigration avec le patriarcat de Moscou au début des années 1920. Contrairement à ce qu'affirme l'auteure, cette Église fut loin d'être majoritaire au sein des communautés émigrées (la géographie de son implantation européenne reste largement à préciser sachant qu'elle eut une audience faible à l'Ouest, en particulier en France). Elle se singularisa par son conservatisme extrême, constituant l'un des terreaux de la radicalisation idéologique de la décennie suivante. De fait, ce fut la seule Église russe émigrée reconnue par le gouvernement nazi. À travers les trajectoires et agissements de ses différents prélats, tel l'évêque Jean de Shanghai, Sheila Fitzpatrick reconstitue 
finement le fil de son engagement politique au cours de l'entre-deux-guerres et jusque dans les camps de personnes déplacées d'après-guerre où elle recruta une nouvelle génération de prêtres.

2 Tout en dressant le noyau historique commun de ces colonies russes blanches, la rétrospective fait une large place à la diversité des milieux émigrés, marqués à Harbin et encore plus à Shanghai par la présence de Juifs russes, eux aussi organisés en diverses associations et qui furent, sans surprise, les cibles d'un antisémitisme croissant au sein des colonies. Cette diversité fut aussi sociale, que l'on saisit à travers l'insertion professionnelle très inégale des émigrés dans les lieux d'accueil. Si les vétérans de Yougoslavie, de Manchourie, ou de la côte chinoise trouvèrent pour partie d'entre eux à s'embaucher dans les troupes de gardes-frontières, de police locale, voire dans des régiments auxiliaires de l'armée japonaise, les destinées furent par ailleurs très contrastées. Les légendaires milieux de perdition de Shanghai trouvent une stupéfiante réalité dans le rapport réalisé à la demande de la Société des Nations en 1935, qui ne dénombre pas moins de 750 prostituées russes professionnelles et plus de 800 occasionnelles : vision de déchéance extrême du monde réfugié russe, handicapé par sa méconnaissance de l'anglais, lingua franca des concessions, qui a en fait surtout vivoté de travaux subalternes avec parfois d'évidentes réussites dans les affaires. Malgré le constat d'un déclassement social assez partagé, certes plus accentué à Shanghai qu'à Harbin, la vie culturelle des communautés extra-européennes fut intense, caractérisée par une presse quotidienne plurielle, l'existence d'établissements russes de qualité, tel le lycée de filles Oksakovskij de Harbin ou l'Institut polytechnique, la notoriété acquise des jazzmen russes de Shanghai, la vivacité des conservatoires de musique, etc. Ces portraits de lieux et de milieux, qui sont aussi ceux de l'espionnage, constituent au fil des pages de vastes fresques sans que pour autant se perde l'un des grands questionnements de l'ouvrage concernant les profils, les affinités politiques des Russes en exil. Face à l'attrait fort du fascisme, très sensible chez les vétérans, mais aussi parmi les jeunes générations, séduites par le modèle mussolinien, d'autres penchants s'observent. White Russians, Red Peril décrit bien des scissions au sein même des colonies entre antibolcheviks et prosoviétiques, qui se manifestèrent tout particulièrement lors des bouleversements politiques et le début de nouvelles migrations. Ainsi, après l'occupation japonaise de la Mandchourie, à partir de 1932, qui entraîna vers le milieu de la décennie, des départs nombreux pour Shanghai, mais aussi des grandes vagues de retours en URSS. Plus de 30000 familles revinrent, qui furent très durement réprimées lors de la Grande Terreur. L'opération menée contre les "Harbincy ", décima 30000 personnes et en entraina près de 20000 dans les camps du goulag. À Shanghai, les sentiments prosoviétiques s'exprimèrent ouvertement à travers la création d'une Union pour le retour, d'un club, alors même que depuis 1937, les Japonais occupaient les lieux, et cette tendance s'accentua avec le déclenchement de la seconde guerre mondiale.

3 L'expérience de la guerre est nécessairement dissociée en Asie et en Europe. La neutralité japonaise face à l'URSS jusqu'en 1943 n'a pas suscité, sinon par la suite et à la marge en Manchourie, d'engagements combattants. L'existence des Russes s'est trouvée parfois favorisée, comme à Shanghai où l'internement des Français et des Britanniques laissa beaucoup de places à prendre. Ceux des Russes qui allaient venir d'Europe vers l'Australie reflétaient, dans bien des parcours, les engagements guerriers aux côtés des armées d'Hitler sous toutes les formes qu'ils aient pris: de l'intégration dans le corps russe de Yougoslavie à l'armée Vlassov et autres forces auxiliaires. La nébuleuse « russe » des 
camps de personnes déplacées (DPs) mêlaient aussi bien des Soviétiques, anciens prisonniers de guerre "retournés» par les nazis, collaborateurs de l'occupant en Ukraine, en Biélorussie, ayant fui lors de sa retraite, anciens travailleurs forcés réfractaires au rapatriement, que des Russes blancs déplacés (de force ou volontairement) des différents pays d'Europe orientale. Derrière cette énumération, une multitude de situations singulières et beaucoup d'autres masquées sous des récits stéréotypés, délivrés lors des procédures d'enregistrement et de vérifications des DPs, qui permettent difficilement d'évaluer la part des anciens collaborateurs actifs, mais entretinrent le soupçon. En Asie, les sympathisants, voire les serviteurs du pouvoir fasciste japonais, connurent des vicissitudes lors de l'entrée en guerre du Japon contre l'URSS, et ce retournement attisa, par ailleurs, un sentiment patriotique prosoviétique latent depuis le début du conflit mondial. En 1946, plus de la moitié des Russes de Shanghai avaient pris la citoyenneté soviétique. Ils furent également nombreux à Harbin à vouloir revenir en URSS, sans toutefois, là, en obtenir le droit jusqu'en 1954. Cette diversité de décisions selon les localisations, inviterait ici à reconsidérer la politique soviétique de rapatriement d'après-guerre moins d'après les lieux de départ qu'à partir du centre moscovite pour éclairer le sens de ces adresses inversées à la vaste diaspora russe.

Celle-ci allait s'accroître avec les nouvelles migrations vers l'Australie, pays en quête de forces laborieuses. Sheila Fitzpatrick analyse les conciliabules menés entre l'office international des réfugiés et le gouvernement australien pour l'accueil des migrants d'après-guerre, soulignant la force de l'antisémitisme régnant parmi les autorités et dans l'opinion australiennes, l'évolution des postures, au rythme de la guerre froide montante, face aux perspectives d'immigration des Russes. À leur égard, les services de surveillance australiennes suspectaient aussi bien les complicités avec l'ennemi d'hier qu'une connivence de cœur avec l'URSS, ce que l'on peut observer dès les premières arrivées qui furent celles des «Européens ». Les Russes fuyant la révolution chinoise, débarquèrent souvent plus tard, au terme parfois de véritables épopées, telle celle de ces milliers de Russes dont l'Organisation internationale des réfugiés avait négocié avec les autorités philippines un accueil transitoire sur l'île de Tubabao avant qu'ils parviennent finalement en Australie, venant renforcer la présence russe blanche en tâchant de pérenniser ses traits d'avant-guerre.

Cette rétrospective dense et compliquée, qui combine une réflexion sur les identités dans leur environnement à la fois extérieur et dans la fabrication du lien communautaire, les suivant dans le mouvement des bouleversements locaux et de la grande histoire, sources de nouveaux déplacements et refondations, relève in fine d'une véritable performance. L'analyse est affranchie de tout jugement sur un sujet comportant pourtant une part d'abîme, tant elle est mobilisée par l'intention primordiale de reconstituer l'univers mental, culturel qui a animé les grands perdants de l'histoire. Elle est le fait d'une spécialiste de l'histoire sociale de l'URSS qui, finalement, est allée explorer, à l'encontre de ses affinités personnelles, souligne-t-elle, les destins de ceux que la révolution avait rejeté sur l'autre bord, les scrutant avec la même impartialité, la même exigence et les mêmes nuances qu'elle avait mises dans ses investigations au cœur du $x^{e}$ siècle soviétique. White Russians, Red Peril représente une contribution substantielle à l'histoire du monde de l'exil russe dans sa longue durée où s'exprime comme un enseignement la grande voix historienne de Sheila Fitzpatrick. 


\section{AUTEURS}

\section{CATHERINE GOUSSEFF}

CNRS - CERCEC 